IJHER International Journal of Humanities and Educational Research ISSN: 2757-5403

Volume 4, Issue 1, February 2022

Received: $20 / 11 / 2021$

Accepted: 06/01/2022

Published: 01/02/2022

\title{
WRITING MULTIFACETED AND PLURAL IDENTITY (FRANCO-IRANIAN) AND THE CONCEPT OF WOMEN'S LIBERATION IN" MARX AND THE DOLL" OF MARYAM MADJIDI
}

\section{Racha Mohamed Hassan EL ENANY ${ }^{1}$}

Dr, 6 October University, Egypt

\begin{abstract}
It is nice to open up to other culture but it is unfortunate that we are uprooted from our cuture.Maryam Madjidi, Iranian writer, she had to leave iran, her home with her family to live in France, to escape from tyranny that prevailed at this time.

Maryam the child, suffered from isolation and living in a strange country using different langage and culture of the Iranian culture and in a struggle between multiple ideas, she has to be forced to live with more than one identity.

To forget her pain, Maryam find in writing the only way to be free.

Our work is based on two parts. To study this novel "Marx And The Doll" , we focus through this study on the impact of internal conflict and plural identity and writing multifaceted with the writer.

Also we care about highlighting on some sustainable development axes about women's liberation and reclaiming her rights to explain the different customs and traditions towards women in Iran from the period of Iranian revolution.
\end{abstract}

Key words: Maryam Madjıd, Marx and The Doll.

http://dx.doi.org/10.47832/2757-5403.12.21 


\title{
L'ÉCRITURE MULTIFORME Á TRAVERS LA MULTI IDENTITÉ (FRANCO-IRANIENNE) ET LA NOTION DE L'ÉMANCIPATION DE LA FEMME DANS "MARX ET LA POUPÉE" DE MARYAM \\ MADJIDI
}

\author{
Racha Mohamed Hassan EL ENANY
}

\section{RÉSUMÉ:}

Il est toujours bon de s'ouvrir aux autres cultures mais il est dommage d'être déraciné de la nôtre.

Maryam Madjidi est une écrivaine iranienne qui a dû quitter son pays natal, avec sa famille, pour vivre en France et s'évader de la gouvernance tyrannique en Iran durant cette période.

Maryam, l'enfant avait subi l'exil, dans un pays étranger avec une langue et une culture différentes à la langue et la culture iranienne.

En conflit entre plusieurs idées, différentes cultures, Maryam se trouve forcée de vivre avec plusieurs identités.

Pour se reconcilier, elle trouve dans l'écriture le seul moyen d'émancipation.

Notre travail se fondera sur deux parties pour l'étude de cette oeuvre,

"Marx et la poupée", On s'appuie sur l'influence du conflit intérieur et la multi- identité de l'écrivaine sur son écriture multiforme.

En plus, nous nous intéresserons à projeter la lumière sur quelques axes du développement durable concernant l'émancipation de la femme et la récupération de ses droits, en montrant les différents rites et coutumes envers la femme en Iran depuis la période de la révolution iranienne.

Mots clés: Maryam Madjid, Marx Et La Poupée.

\section{Introduction}

La Patrie est l'endroit où l'homme nait, existe, grandit et crée pour évoluer. C'est la notion à laquelle chacun appartient, se sent attiré et lié mais parfois elle nous rappelle des souvenirs malheureux.

Maryam Madjidi raconte ses souvenirs en étant en conflit entre son appartenance à son pays et son obligation à la soumission à l'idée de l'évasion de toute sa famille vers la France.

L'écrivaine se trouve obligée d'exclure progressivement son identité iranien, sa langue et sa personnalité pour d'autres français.

La narratrice trouve dans l'écriture le moyen d'évasion par lequel elle cherche le repit pour transmettre au monde son cri de femme résignée. Sa multi identité influence sa pensée et son écriture multiforme.

On remarque à chaque phrase de Maryam Madjidi une hybridité d'identité qui apparait à travers les descriptions des rites, des coutumes des pays visités et les dialogues échangés.

Maryam commence son récit d'une manière tout à fait différente. Elle nous projette son image dans le ventre de sa mère. Elle nous transporte vers la vie iranienne, les combats, les conflits et les espoirs d'émancipation.

C'est pourquoi, notre travail se formera de deux parties, dans la première partie on traitera les différentes forme d'écriture où l'écriture multiforme dans "Marx et la Poupée" et dans la deuxième partie, on montrera ses essais d'émancipation comme l'un des axes du développement durable. 


\section{La première partie:}

\section{La Multi identité et l'écriture multiforme}

Vivant en iran depuis son enfance, Maryam Madjidi l'écrivaine de notre oeuvre "Marx et La Poupée", commence son écriture par l'expression de la relation intime qui peut lier entre l'homme et son pays natal.

"La patrie n'est qu'un compartiment dans le désert" (Proverbe tibétain)

Maryam décrit les peines, les chagrins et les déchirements vécus par ses parents durant leur présence à Téhéran. Les menaces, la torture et la mort les entourent partout. La tyrannie du pouvoir iranien durant cette période des années 1980 et la violence des autorités obligent le peuple à s'évader; même les révolutionnaires "sentent leur foi(...) décliner" (Madjidi, 2017, p. 42)

La peur entourent tous les iraniens, ils perdent la paix et leur droit de liberté :

"Ce pays massacre ses meilleurs enfants." (Madjidi, 2017, p. 39)

Maryam, la petite fille, tout en jouissant entre sa grand mère qui lui offre beaucoup de jouets et ses parents qui la gate, elle se trouve obligée de quitter tout son environnement pour vivre à l'étranger, dans un nouveau pays.

"Paris est étouffant pour elle. La France entière." (Madjidi, 2017)

Entre ces mauvaises circonstances, Maryam la petite fille est tiraillée, en conflit entre plusieurs idées. Une révolution intérieure continue à déchirer Maryam l'enfant, l'adolescente et la jeune écrivaine qui trouve dans l'écriture le moyen d'expression de ses sentiments contadictoires. Ceci se traduit à travers les différentes formes d'écriture narratives, littéraires, poétiques et épistolaire rassemblées dans "Marx et la poupée".

\section{Le récit narratif et poétique}

Au commencement, Maryam écrit un récit narratif, elle relate l'histoire depuis sa présence comme embryon dans le ventre de sa mère, observe les évènements, suit sa mère en espérant de la protéger.

"Je me recroqueville un peu plus au fond du ventre" (Madjidi, 2017, p. 12)

La première page de "Marx et la Poupée" est un poème intitulé" La Pierre" et décrit l'histoire d'un homme qui est entrain de prévoir l'arrivée d'un bébé, une fille qui s'appelle Maryam.

L'écrivaine essaie d'intégrer le style poétique avec le style narratif comme si elle veut soulager sa douleur apparante dans son récit et l'embellir par des vers en langue persane et française.

"Il est un profond secret qu'il ne faut pas dire aux profanes:

La tulipe qui se fane ne refleurira jamais" (Madjidi, 2017, p. 77)

Une quête identitaire apparait dans le récit de Maryam et ceci est souligné par l'intégrétion de certains vers iranien dans son récit.

Tout au long du roman de Madgidi, on trouve en parallèle le récit narratif et poétique.

La narratrice veut lier entre la fiction et la communication, comme si elle veut donner aux mots certaines signes exposées aux décodage du lecteur.

Selon Gérard Genette: "le récit ne représente pas une histoire réelle ou fictive, il la raconte c'est à dire qu'il la signifie par le moyen du langage. (Genette, 2021)

\section{Le récit d'évènements et le récit de parole:}

$\mathrm{Au}$ fur et à mesure que Maryam décrit les évènements de la révolution iranienne (Madjidi, 2017, p. 35)"le cauchemar "qui les a toujours suivi

elle s'intéresse à reprendre même les paroles "-Mais bon sang, qu'est ce qu'on a fait pour avoir une enfant pareille! Elle ne pige rien au communisme" ce sont les paroles de la mère de Maryam qui veut sa fille communiste comme eux. 


\section{Le récit épistolaire:}

Parfois, Maryam Madjidi utilise le style épistolaire pour expliquer ses idées à travers des dialogues entre elle et d'autres personnes ou en représentant les évènements à travers les correspondances.

"maman, où est papa?

Dans un autre pays.

-C'est quoi un autre pays

-ça s'appelle la France."

(Madjidi, 2017, p. 44)

L'écrivaine de "Marx et la Poupée " rassemble entre les différents genres d'écriture dans une seule oeuvre, comme si elle veut exprimer les divers sentiments qui la déchirent, les différents pays où elle vit et les multiples identités qu'elle reçoit.

"les conditions de vie d'un être vivant sont liées à de nombreux facteurs"

(Jacques, 2018, p. 83)

\section{Le recours à l'intertextualité:}

Parfois Madjidi a recours à l'intertextualité pour exposer sa culture iranienne et la langue persane. Elle recite dans certaines pages des vers de Omar Khayyâm:

"مهتاب به نوردامن شب بشكافت" (Madjidi, 2017, p. 75)"

De temps en temps, l'écrivaine mentionne quelques éléments de la vie en Iran: "Miami party", le "Jordan street"

(Madjidi, 2017, pp. 64, 67)

\section{Le recours à l'ekphrasis:}

Parmi les parties de l'intertextualité dans l'oeuvre de Maryam Madjidi est l'ekphrasis qui représente la description d'oeuvres artistiques

Mariam rappelle ses lecteurs certaines oeuvres artistiques comme "les tableaux de Delacroix" (Madjidi, 2017, p. 74)

\section{La mention chronologique et cosmique:}

Tout en parcourant les champs de ses souvenirs, la narratrice mentionne à chaque partie le temps- la ville et l'espace.

Mariam essaie à travers la reprise du temps et l'espace de reprendre les évènements et de les situer. Elle aide son lecteur à mieux suivre ses différents déplacements et sentir ses différentes émotions.

"1980-université de Téhéran" (Madjidi, 2017, p. 12)

"2012- téhéran- Dans un taxi" (Madjidi, 2017, p. 198)

\section{Des titres exceptionnels:}

Maryam madjidi excelle même dans le choix des titres exceptionnels pour chaque chapitre de son oeuvre. Les titres nous invitent à mieux comprendre et prévoir les tiraillements qui torturent les gens durant cette période.

" il était une fois dans le ventre de la mère-fantôme sans bouche-Nouchâbé -L'enfant du parti- La peur- il était une fois les mains du père- comment peut -on être persane....". (Madjidi, 2017)

\section{Une répartition différente}

La narratrice de Marx et la poupée intitule les différentes parties de son oeuvre d'aprés son déplacement d'un pays à l'autre et toujours dans une quête d'identité.

La Première naissance concerne sa vie avec sa famille dans son pays natal en Iran.

La deuxième naissance est son voyage avec ses parents pour vivre en France. 
La troisième naissance est le retour de l'écrivaine à sa patrie pour reprendre tout ce qui était perdu au fil des années.

Et pour chaque station de sa vie c'est une nouvelle naissance qui lui laisse beaucoup d'expériences et de souvenirs.

La narratrice insiste enfin à retourner à son pays, s'évader de son déracinement et son isolement malgré qu'elle trouve les mêmes idées fanatiques.

Maryam cite les mots de sa mère en essayant de la convaincre de retourner en France:

" Tu veux être considérée comme une sous-merde parce que tu as un vagin ?" (Madjidi, 2017, p. 191) ce qui figure le regard de l'homme aux femmes en Iran.

\section{L'image de la société à travers l'écriture}

Dans des conditions difficiles,"le désarroi ou l'inconscience" (Weil, 1955) Madjidi trouve qu'il est trés important d'esquisser les différents problèmes de la société et de les transmettre, peut-être par l'écriture multiforme pour bien les concrétiser.

On trouve sur certaines pages les slogans des manifestations " ils nous ont volé notre révolution.

Ils nous ont volé notre démocratie.

Ils nous ont volé notre vote."

(Madjidi, 2017, p. 59)

L'écrivaine expose parfois son état psychique, durant son enfance, qui représente l'image des filles iraniennes durant cette ère " la terreur qui m'habitaient"

(Madjidi, 2017, p. 107)

De temps à l'autre, la narratrice cite des proverbes ou des poèmes empruntés à d'autres écrivains pour décrire le désespoir et les malheurs des années de la révolution iranienne.

"Et tu mourras tout en sachant que rien n'est plus beau, que rien n'est plus vrai que la vie" Nâzim Hikmet (Madjidi, 2017, p. 10)

\section{L'usage des pronoms personnels}

Tout en cherchant à sauver ses souvenirs du relâchement, l'écrivaine a recours au récit transpersonnel.Elle réfère à plusieurs voix dans sa narration dans un monologue intérieur, elle passe de la première personne du singulier à la deuxième personne "tu" et puis elle retourne au pronom toi comme pour expliquer qu'elle est en train de relater son autobiographie, représentant la collectivité.

Elle poursuit un monologue intèrieur, raconte ses confessions ou s'adresse à sa mère dans un cadre socio-historique.

Maryam Madjidi intègre dans son récit des procédés picturaux qui aident le lecteur à vivre la révolution iranienne, l'obligation à l'immigration, le sentiment d'ètre étranger, la quête d'identité entre plusieurs langues, pays, rites et coutumes.

"tu as creusé un trou en moi dans lequel toutes les angoisses de ma vie future prendront racine" (Madjidi, 2017, p. 14)

L'écrivaine de "Marx et la poupée" se trouve condamnée à quitter l'iran avec sa famille, vivre en France et en Istanbul sans jamais oublier son pays natal.

D'aprés Philippe Haeck :

"l'écrivain prône une contribution importante de l'art à l'histoire de notre société, à son devenir plus humain surtout. (Roy, 2021)

\section{Le choix des verbes significatifs:}

Même le choix des temps verbaux aborde le mal vécu, la violence et l'oppression.

" hurlent", "fouillent", "brise", "tremblent", "arrachent". (Madjidi, 2017, p. 13) 
La narratrice relate ses témoignages par rapport au milieu social où elle vit et les histoires racontées par les parents et les amis.

\section{Deuxième Partie}

\section{La notion De L'émancipation De La Femme}

En traitant les chroniques de la vie à Téhéran, Maryam Madjidi touche l'un des axes du développement durable, c'est l'émancipation de la femme.

" l'inégalité des pouvoirs et les restrictions d'accés fondées sur le sexe, l'appartenance ethnique, la richesse et autres differences peuvent bloquer l'insertion sociale de certaines communautés et favoriser les discriminations." (ZANA, 2009, p. 34)

Maryam Madjidi cherche à travers son univers, son entourage iranien, à libérer les femmes du monde entier, la libération de l'expression, de la pensée, des mauvaises coutumes comme l'excision....etc.-

L'écrivaine veut trouver "un monde meilleur, un avenir positif pour les générations futures, allégé des dettes environnementales, sociale et économique." (Attali, 2018, p. 189)

\section{La violence à l'égard de la femme:}

Maryam Madjidi, présente les contraintes des téhéranaises, leurs témoignages, la torture qu'elles subissent. Les frontières mises par la société iranienne, empêchant la persane de toute liberté "des intellectuels post-islamistes se font les porte-parole de la société " (Roussillon, 2018)

L'écrivaine intégre des images des crimes commis sur les femmes durant ce temps. Au debut de l'oeuvre, elle maintient sa posture de narratrice qui témoigne tout ce qui se déroule autour d'elle en étant dans la ventre de sa mère.

"Les voiles des femmes sont piétinés; les mains arrachent leurs cheveux. Les femmes sont trainées par terre, elles se débattent comme elles peuvent et les hommes les traitent de sales putes." (Madjidi, 2017, p. 13)

La femme n'a pas le droit de se révolter, de recvoir leurs droits, ni même de se porter selon leur volonté. p. 40)

"les iraniennes non voilées sont molestées par les miliciens islamiques" (Minoui, 2009,

\section{Le terrorisme et le fanantisme religieux:}

La peur habite toutes les maisons iraniennes, les gens ne montrent pas leurs croyances de craintes d'être violés et prisonnés.

"ces hommes ont les yeux injectés de sang et brandissent des batons plantés de clous"

"le bruit d'un crane qu'on brise" (Madjidi, 2017, p. 13)

L'affolement et le tremblement entre les gens en Iran durant ce temps, les empêchent de vivre ou de préserver leur liberté. Malgré leurs luttes, toutes les réformes sont en vain.

" c'est la menace d'un régime de plus en plus autoritaire" (Todd, 2020, p. 11)

\section{La souffrance des mères iraniennes:}

La narratrice de "Marx et la poupée " trace les causes qui poussent sa famille à s'évader et quitter leur pays natal. Elle explique comment les parents perdent chaque jours des membres de leurs familles dans les actes de résistance. Envahi par la panique, le père Madjidi décide l'immigration.

La mère, le père, la grand mère et même les amis de la famille Madjidi représentent les témoins qui racontent les évènements sans falsifications.

Surtout les mères irannienne souffrent des malheurs de la révolution. Elles perdent leurs fils, leurs maris et leurs frères. L'écrivaine raconte des actes creusés dans sa mémoires 
des jeunes hommes attaqués. Elle cite parfois des noms "la mère d'Abbas". (Madjidi, 2017, p. 30) qui pleure toujours après avoir perdu son fils. La mère décrit l'atrocité et la cruauté des agents de police en attrappant son fils, comment ils l'arrachent du lit sans même s'habiller jusqu'à sa mort enfin. prison.

Même, la petite Maryam fait la queue avec sa grand mère pour visiter son oncle au

\section{La repression des jeunes persanes}

Depuis leur enfance, les filles persanes vivent les malheurs de la période révolutionnaire, Maryam à l'âge de cinq ans se trouve obligée de laisser tous ses jouets et de les donner aux autres enfants de son quartier pour partir en France avec ses parents. La petite fille a eu une dépression et pense à enfouir ses poupées au jardin.

Les adolescents ont peur de sortir. Elles n'ont pas le plaisir de porter les jeans ou les autres vêtements des jeunes, elles doivent suivre les règlements et refuser les traits de la culture occidentale.

Les jeunes persanes n'arrivent pas à s'intégrer dans le nouveau régime, elles sont dans une quête d'identité.

"Dehors on pleure, Dedans on rit. Dehors, on rit. Dehors, on se recouvre du voile obligatoire. Dedans, on exhibe son nombril, entre un T-shirt trop court et un bleu-jeans taille basse. Pas facile de s'y retrouver" (Minoui, 2009)

La repression génère chez plusieurs persanes l'explosion, beaucoup de jeunes filles veulent faire ce qui est defendu " le concept; porter un bikini pour les filles(...)on est tous autour d'une grande piscine, on boit des cocktails ou du champagne, on fume de l'herbe, on danse et on sauté dans la piscine." (Madjidi, 2017, p. 64)

Même les femmes iraniennes mariées durant cette période, subissent la torture et l'engagement :

"il m'a giflée et trainée par les cheveux(...) il m'a frappé encore au visage(...) tu n'es rien sans rien sans moi, tu n'es qu'une femme" (Madjidi, 2017, p. 67)

La femme iranienne de cette période n'a pas le droit de s'abstenir ou de s'objecter, elle n'a qu'accepter ce que son mari lui demande.

\section{La recherche de l'émancipation à travers les différentes identités}

Le monde européen représente aux iraniens un monde étranger sombre, mystérieux et étonnant. Ils sortent de leur pays en souhaitant d'y retourner en trouvant la femme émancipée.

Maryam en décrivant ces premiers jours en France, elle parle même de la nourriture française comme aliment sans goût ni saveur: Je regarde ces croissants posés tristement sur la table" (Madjidi, 2017, p. 96)

A l'âge de 32 ans et en vivant en chine, l'écrivaine cherche l'odeur de ce croissant français refusé au début de sa présence en France. Maryam est toujours en quête d'émancipation et d'identité, elle cherche la stabilité.

Même lorsque Maryam enfouie ses jouets, elle ne cherche pas seulement à les cacher des autres enfants pauvres de la rue mais elle cherche à les émanciper du monde tyranique où elle vit dans l'intention de les reprendre un jour dans une société plus civilisée. 


\section{L'émancipation de la femme musulmane}

Maryam discute dans son oeuvre le sujet des musulmans installés dans un pays occidental. Les gens traitent les musulmans surtout les musulmanes portant le voile comme terroristes ou des enemies qui les menacent malgré qu'ils ne sont pas fanatiques.

L'auteure décrit ces premiers jours à l'école, les regards de ses copines, leur éloignement d'elle. Elle se rappelle toujours de sa solitude à Paris au commencement de leur installation même elle a peur de leur dire ses origines.elle se sent exilée.

Maryam veut corriger par son oeuvre l'idée des étrangers envers la femme musulmane, elle veut l'émanciper de ces pensées qui s'opposent à la réalité.

" L'installation des musulmans en terres occidentales suscite énormement des reactions souvent négatives. Une notion aussi utilisée que controversé, l'islamaphobie" (Bachir, 2019, p. 20)

L'écrivaine relate la réalité, elle comble son conflit par l'écriture en espérant de trouver un jour le remède et dire " le pouvoir ne se résume pas à la domination" (Bozarslan, 2014, p. 53)

\section{Conclusion}

"la patrie n'est qu'un campement dans le désert" (Madjidi, 2017, p. 10)

" Je suis une guirlande de mots accrochée à un arbre qu'un enfant montre du doigt." (Madjidi, 2017, p. 202)"

Maryam Madjidi se livre à ses souvenirs et si on relie entre la première et la dernière phrase de Maryam Madjidi dans son oeuvre "Marx et la poupée"; On peut comprendre du proverbe cité au commencement du livre qu' elle explique dans son oeuvre que malgré le sentiment amer de l'isolement, le pays étranger devient enfin familier mais la patrie continue à être enfouie dans le coeur.

A travers la dernière phrase de l'écrivaine, on peut déduire qu'après de profondes reflexions dans sa quête de soi, Maryam Madjidi fait appel à son inconscient. Elle fait revivre l'histoire pour l'exposer aux lecteurs et leurs donner libre cours pour comprendre la réalité. Le choix du mot guirlande n'est qu'une périphrase pour expliquer l'enchainement des évènements enfouis dans la mémoire et relatés par la narratrice mais sans trouver la solution.

La narratrice traite plusieurs sujets concernant la vie iranienne, sa beauté, son charme comme pays natal et son atrocité par le terrorisme ou l'injustice qui s'y trouvent.

Maryam Madjidi cherche à symboliser par sa mémorisation des différentes stations de sa vie, toute sa génération. Téhéran, de l'ère de la révolution, ce pays qui "vue de l'extérieur, fait peur" (Minoui, 2009) n'est qu'un pays victime de certains règlements qui surtout privent la femme de sa liberté d'expression.

Malgré son éloignement de son pays pendant des années, la narratrice rêve du retour et le réalise en rêvant toujours du changement " ça va changer, je suis sûre" (Madjidi, 2017, p. 199) 


\section{Bibliographie}

Attali, J. (2018). Positive Planet- vers une révolution positive. france: Fayard.

Bachir, R. B.-S. (2019). La controverse dialogue sur l'islam. stock.

Bozarslan, H. (2014). Le luxe et la violence. Paris: CNRS.

Genette, $\quad$ G. $\quad(2021, \quad 11 \quad$ Samedi). $\quad$ Retrieved from http//www.signosemio.com/genette/narratologie.asp.

A. Jacques. Positive planet. In. Fayard(2018).

In M. Madjidi. Marx et la poupée.. Le Nouvel Attila(2017).

In D. Minoui. Jacob-Duvernet. Les pintades à Téhéran (2009)

(n.d.). Proverbe tibétain. In Marx et la Poupée (p. 9).

.In A. Roussillon, Réforme et politique dans le monde arabe. Paris: CNRS(2018).

Roy, M. (2021, $10 \quad$ 22). $\quad$ erudit.org. Retrieved from https://www.erudit.org/en/journals/vi/1900-v1-n1-vi1402/200280ar.pdf.

.In E. Todd.Les luttes de classes en France au XXIe siècle. Paris: Seuil(2020).

Weil, S. (1955). Réflexions sur les causes de la liberté et de l'opression sociale. Paris: Gallimard.

ZANA, P. (2009). 50 mots pour comprendre le développement durable. Paris: Alternatives. 